\title{
Study of the Fabrication of BCB Waveguide Devices by Ultraviolet Pulsed-Laser Illumination
}

\author{
Wan-Shao Tsai, Student Member, IEEE, Liang-Yin Chen, Student Member, IEEE, and \\ Way-Seen Wang, Member, IEEE
}

\begin{abstract}
Benzocyclobutene directional couplers and $1 \times 2$ multimode interference (MMI) power splitters fabricated by ultraviolet (UV) pulsed-laser illumination are presented. With an accurate control of the fabrication process, the measured coupling lengths of the directional couplers are in good agreement with those simulated by the beam propagation method. In particular, accurate power splitting ratios of the directional couplers can be achieved by controlling the number of laser shots to obtain the required index changes. Moreover, MMI power splitters with accurate interference lengths and low imbalances can be successfully fabricated. All those reveal that UV pulsed-laser illumination is an accurate and controllable technique for the device fabrication.
\end{abstract}

Index Terms-Benzocyclobutene (BCB), excimer lasers, optical polymers, optical waveguides.

\section{INTRODUCTION}

$\mathbf{O}$ PTICAL waveguides using polymer materials are attractive in recent years due to their low cost and simple fabrication process [1], [2]. Among various polymer materials, benzocyclobutene $(\mathrm{BCB})$ has been widely used in multilayer interconnection and optical waveguides for its versatility of material properties, such as low moisture absorption, low optical loss, and high chemical resistance [3], [4]. Furthermore, BCB is strongly absorbed at wavelengths shorter than $300 \mathrm{~nm}$ [5]. Therefore, krypton fluorine (KrF) excimer laser illumination on BCB film has been successfully used for the fabrication of buried-type BCB waveguides [6]. To demonstrate the application of the ultraviolet (UV) pulsed-laser illumination technique, waveguide devices such as directional couplers and $1 \times 2$ multimode interference (MMI) power splitters are fabricated in this work. As both devices are known to be sensitive to the device and process parameters, the coupling length of a directional coupler and the MMI region of an MMI power splitter must be carefully designed and fabricated in order to have desired device characteristics. In this work, the UV pulsed-laser illumination technique is used to adjust of the index change by controlling the number of laser shots. The laser adjustable index change provides a flexible control over the power splitting ratio of directional coupler with a fixed coupling length. Moreover, MMI

Manuscript received July 10, 2007; revised October 7, 2007. This work was supported by the National Science Council, Taipei, Taiwan, R.O.C., under Contract NSC 95-2221-E-002-326-MY3.

The authors are with the Graduate Institute of Electronics Engineering, National Taiwan University, Taipei, Taiwan 106, R.O.C. (e-mail: f91943030@ ntu. edu.tw; f91943031@ntu.edu.tw; wswang@cc.ee.ntu.edu.tw).

Digital Object Identifier 10.1109/LPT.2007.912560

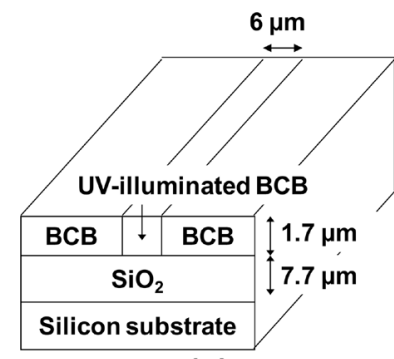

(a)

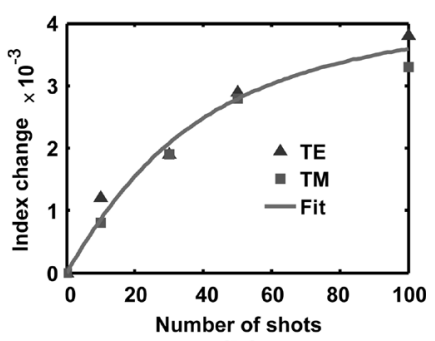

(b)
Fig. 1. (a) Device structure of buried-type BCB waveguide. (b) Refractive index change versus the number of laser shots.

power splitters with accurate interference lengths and low imbalances can also be successfully fabricated. Thus, good agreement between the simulated and experimental results can be found.

\section{EXPERIMENT}

A schematic diagram of the UV-illuminated BCB waveguide is shown in Fig. 1(a). First, a silicon oxide $\left(\mathrm{SiO}_{2}\right)$ cladding layer of thickness $7.7 \mu \mathrm{m}$ is grown on the silicon substrate by plasma-enhanced chemical vapor deposition. A BCB layer of thickness $1.7 \mu \mathrm{m}$ is then spin-coated on top of the silicon oxide. After soft curing at $90^{\circ} \mathrm{C}$ for $20 \mathrm{~min}$, the film is thermally cured in a nitrogen flowing furnace at $250^{\circ} \mathrm{C}$ for $1 \mathrm{~h}$ and then cut into suitable sizes for UV-illumination. The UV source is a $\mathrm{KrF}$ excimer laser of wavelength $248 \mathrm{~nm}$, with a beam size of $3 \mathrm{~mm}$ $\times 6 \mathrm{~mm}$ and an energy density of $100 \mathrm{~mJ} / \mathrm{cm}^{2}$. As before, the pulse repetition rate is controlled at $5 \mathrm{~Hz} \mathrm{[6]} \mathrm{and} \mathrm{the} \mathrm{laser} \mathrm{beam}$ size is then expanded by a fused silica focusing lens such that the UV laser pulses can be uniformly illuminated on the sample through a quartz-chromium mask. For numerical simulation, the refractive indexes of $\mathrm{BCB}$ (before illumination) and $\mathrm{SiO}_{2}$ layer at $1548 \mathrm{~nm}$, measured by the prism coupler (Metricon 2010), are 1.5484 and 1.4540 , respectively.

Previous experimental results showed that the index change is linearly increased with the number of laser shots and then tends to be constant when the number of laser shots is greater than 300 . To prevent the $\mathrm{BCB}$ film from being seriously damaged, less than 100 laser shots were chosen for the fabrication of optical waveguides [6]. That indicates the range of index change should be chosen as $0.001-0.004$, which corresponds to $10-100$ laser shots, as shown in Fig. 1(b). The index profile is verified as step-like with 11 modal effective indexes measured by the prism coupler. 


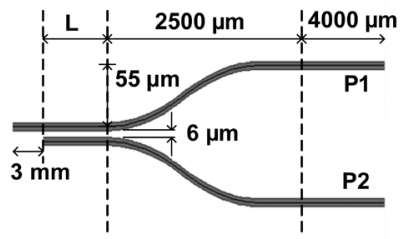

(a)

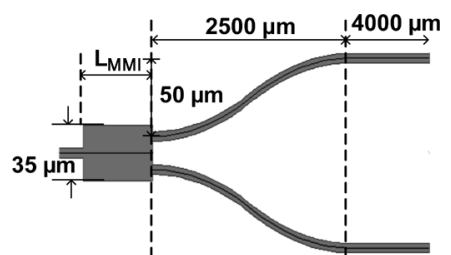

(b)
Fig. 2. Top view of designed (a) directional couplers and (b) $1 \times 2$ MMI power splitter.

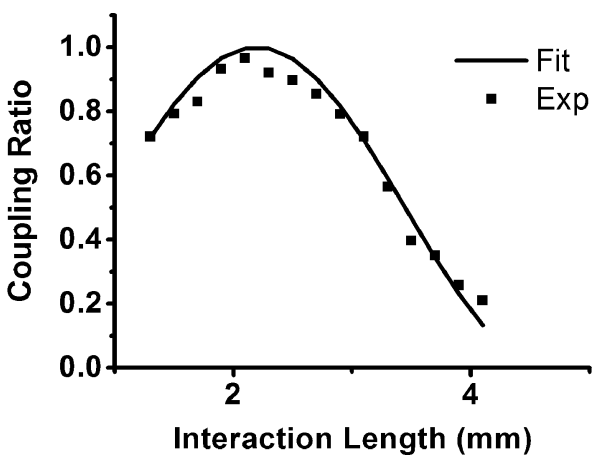

Fig. 3. Coupling ratios of directional couplers with various interaction lengths for an index change of 0.0033 .

For characterization, the waveguide devices are launched with a laser of wavelength $1548 \mathrm{~nm}$ by end-fire coupling. The output field intensity contours are measured by a vidicon camera and analyzed by a beam analyzer. And the output power is detected by a power meter. The average propagation loss at $1548 \mathrm{~nm}$ of channel waveguides fabricated with less than 100 laser shots is $0.6 \mathrm{~dB} / \mathrm{cm}$ with an error margin of $\pm 0.2 \mathrm{~dB} / \mathrm{cm}$.

\section{A. Directional Couplers}

The designed layout of directional couplers is shown in Fig. 2(a). For a single guiding mode at $1548 \mathrm{~nm}$, the waveguide width is chosen as $6 \mu \mathrm{m}$, and an S-bend is applied in the curvature region. To find the coupling length $L_{c}$, directional couplers of various interaction lengths $L$, varying from 1.3 to $4.1 \mathrm{~mm}$, were fabricated. The measured coupling ratio versus the interaction length of a directional coupler with an index change of 0.0033 is shown in Fig. 3, where the coupling ratio is defined as $P_{2} /\left(P_{1}+P_{2}\right)$, and $P_{1}$ and $P_{2}$ are the output power of the input and the coupled branches as indicated in Fig. 2(a). The experimental results can be fitted to a function of the form $\sin ^{2}(\kappa L+\phi)$, where $\kappa$ is the coupling coefficient and $\phi$ is the phase shift due to the interaction near the branching areas [7]. The fitted values of $\kappa$ and $\phi$ are 0.6278 and 0.1933 , respectively. The value of $L_{c}$ is then calculated as $2502 \mu \mathrm{m}$. As compared with the value of $L_{c}$ simulated by the beam propagation method $(2507 \mu \mathrm{m})$, the percentage error is below $1 \%$. This shows that the proposed UV-illumination technique is quite accurate in the fabrication of the BCB waveguide devices.

To demonstrate flexible control over the waveguide devices by the UV-illumination technique on BCB films, directional couplers with a fixed interaction length but different power splitting ratios are fabricated. Index changes, power splitting ratios,
TABLE I

Index Change, Power Splitting Ratio, AND TRAnSmission EFFiciency of THE PROPOSED DiRECTIONAL COUPLER

\begin{tabular}{|c|c|c|c|c|c|c|c|}
\hline \multirow{3}{*}{$\begin{array}{l}\text { Index } \\
\text { change }\end{array}$} & \multicolumn{3}{|c|}{ Experiment } & \multicolumn{3}{|c|}{ Simulation } & \multirow{3}{*}{$\begin{array}{c}\text { Abs. } \\
\text { error } \\
\text { of } P_{1} \\
(\text { or } \\
\left.P_{2}\right) \\
(\%)\end{array}$} \\
\hline & \multicolumn{2}{|c|}{$\begin{array}{c}\text { Output power } \\
(\%)\end{array}$} & \multirow{2}{*}{$\begin{array}{c}\text { Trans. } \\
\text { effic. } \\
(\%)\end{array}$} & \multicolumn{2}{|c|}{$\begin{array}{c}\text { Output power } \\
(\%)\end{array}$} & \multirow{2}{*}{$\begin{array}{c}\text { Trans. } \\
\text { effic. } \\
(\%)\end{array}$} & \\
\hline & $P_{1}$ & $P_{2}$ & & $P_{1}$ & $P_{2}$ & & \\
\hline 0.0024 & 27.23 & 72.77 & 81.2 & 27.85 & 72.15 & 83.4 & 0.62 \\
\hline 0.0028 & 37.85 & 62.15 & 89.7 & 37.39 & 62.61 & 91.3 & 0.46 \\
\hline 0.0031 & 43.88 & 56.12 & 94.1 & 45.33 & 54.67 & 96.8 & 1.45 \\
\hline 0.0033 & 50.95 & 49.05 & 95.8 & 49.77 & 50.23 & 97.4 & 1.18 \\
\hline
\end{tabular}

Interaction length: $19 \mu \mathrm{m}$

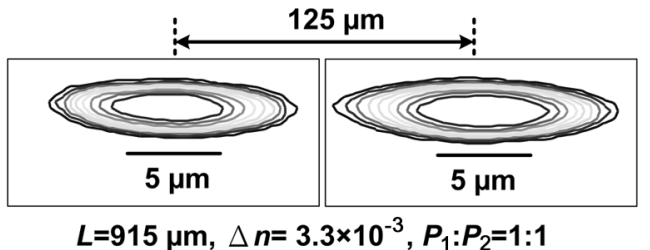

(a)

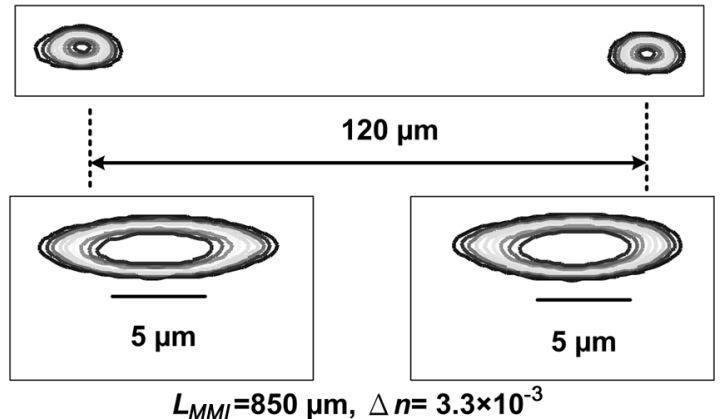

(b)

Fig. 4. Field intensity contours of (a) the directional couplers and (b) the $1 \times$ 2 MMI.

and transmission efficiencies of the proposed directional couplers are listed in Table I. With a fixed interaction length of $915 \mu \mathrm{m}$, the power splitting ratio can be adjusted from $1: 2.7$ to $1: 1$ when the index change is varying from 0.0024 to 0.0033 by the UV-illumination. The measured transmission efficiencies are all higher than $81 \%$, which are very close to those simulated. In particular, Fig. 4(a) shows the intensity contours of the directional coupler with an index change of 0.0033 for equal power splitting. As can be seen from the figure, the intensity contours are uniformly spaced, which indicates the UV pulses have been uniformly expanded for a stable illumination.

Moreover, as indicated in Table I, the measured power splitting ratios are in good agreement with the simulated ones. Note that the absolute errors of the output power $P_{1}$ (or $P_{2}$ ) are all below $1.5 \%$. The accurate power splitting ratios indicate the feasibility of a stable control on the refractive index change by adjusting the number of laser pulses.

\section{B. MMI Power Splitters}

The MMI power splitter has been known for its compact size and low imbalance as a power-splitting device [8]. The use of 
TABLE II

TRANSMISSION EFFICIENCIES AND THE IMBALANCE OF MMI CORRESPOND TO INTERACTION LENGTHS

\begin{tabular}{ccccc}
\hline \hline \multirow{2}{*}{$\begin{array}{l}L_{M M I} \\
(\mu \mathrm{m})\end{array}$} & \multicolumn{2}{c}{ Experiment } & \multicolumn{2}{c}{ Simulation } \\
\cline { 2 - 4 } & $I(\mathrm{~dB})$ & \multicolumn{2}{c}{$T(\%)$} & $\begin{array}{c}\text { Percentage } \\
\text { error of } T(\%)\end{array}$ \\
\hline 700 & 0.33 & 78.88 & 80.10 & 1.52 \\
750 & 0.05 & 84.58 & 86.14 & 1.81 \\
800 & 0.18 & 87.71 & 90.53 & 3.12 \\
850 & 0.06 & 92.03 & 92.52 & 0.53 \\
900 & 0.01 & 89.96 & 91.86 & 2.07 \\
950 & 0.07 & 86.00 & 88.68 & 3.02 \\
1000 & 0.05 & 81.57 & 83.60 & 2.43 \\
\hline \hline
\end{tabular}

polymer materials for weak-guiding MMI devices has been reported previously [2], [9]. In this work, weak-guiding MMI power splitters are also fabricated by UV-illumination for comparison. The designed layout of a $1 \times 2$ MMI power splitter is shown in Fig. 2(b). The index change is chosen as 0.0033 . First, MMI power splitters with an interaction length $L_{\mathrm{MMI}}$ varying from 700 to $1000 \mu \mathrm{m}$ are fabricated. To evaluate the performance of the MMI power splitter, the power transmission efficiency, defined as $T(\%)=P_{\mathrm{MMI}} / P_{\mathrm{str}}$, where $P_{\mathrm{MMI}}$ and $P_{\text {str }}$ are the sum of the two output power and that of the straight waveguide, is measured. Moreover, the imbalance of the devices, defined as $I(\mathrm{~dB})=10 \log \left(P_{\max } / P_{\min }\right)$, where $P_{\max }$ and $P_{\min }$ are the maximum and minimum output power, is also measured. The power transmission efficiencies of the MMI power splitters corresponding to different interaction lengths are listed in Table II. As indicated in the figure, the highest transmission efficiency is $92.03 \%$ when the interaction length is $850 \mu \mathrm{m}$. The simulated transmission efficiency calculated with the same interaction length is $92.52 \%$. The percentage error of $T$ is only $0.53 \%$ upon comparison. Moreover, the measured imbalance is $0.06 \mathrm{~dB}$, which is slightly larger than the ideal one $(0 \mathrm{~dB})$. The corresponding output power intensity contours are shown in Fig. 4(b). As can be seen from the figure, both output intensity contours are nearly the same. For further comparison, the power transmission efficiencies correspond to other interaction lengths and the imbalances are also listed in Table II. As can be seen, the averaged imbalance is $0.11 \mathrm{~dB}$ and the averaged error of transmission efficiency is $2.07 \%$. Good agreement between the experimental and the simulated results shows that the fabrication process can be accurately controlled, which is potentially useful for device design and fabrication.

\section{CONCLUSION}

BCB directional couplers and MMI devices were successfully fabricated by UV pulsed-laser illumination. The measured coupling lengths of the directional couplers and the interference lengths of the MMI power splitters are in good agreement with those simulated. Furthermore, the power splitting ratios of the directional couplers can be adjusted by controlling the numbers of laser shots. Experimental results show that directional couplers with high transmission efficiencies for wide range of power splitting ratios can be successfully fabricated. Moreover, MMI power splitters with high transmission efficiencies and low imbalances can also be achieved. The accurate control of the UV pulsed-laser illumination technique is potentially usefully for the design and fabrication of other polymer waveguide devices. Details of the application of the proposed UV pulsed-laser illumination will be of great interest in the future.

\section{REFERENCES}

[1] L. Eldada, C. Xu, K. M. T. Stengel, L. W. Shacklette, and J. T. Yardley, "Laser-fabricated low-loss single-mode raised-rib waveguiding devices in polymers," J. Lightw. Technol., vol. 14, no. 7, pp. 1704-1713, Jul. 1996.

[2] D. G. Rabus, P. Henzi, and J. Mohr, "Photonic integrated circuits by DUV-induced modification of polymers," IEEE Photon. Technol. Lett., vol. 17, no. 3, pp. 591-593, Mar. 2005.

[3] C. F. Kane and R. R. Krchnavek, "Benzocyclobutene optical waveguides," IEEE Photon. Technol. Lett., vol. 7, no. 5, pp. 535-537, May 1995.

[4] K. P. Lor, Q. Liu, and K. S. Chiang, "UV-written long-period gratings on polymer waveguides," IEEE Photon. Technol. Lett., vol. 17, no. 3 , pp. 594-596, Mar. 2005

[5] Y. G. Zhao, W. K. Lu, Y. Ma, S. S. Kim, and S. T. Ho, "Polymer waveguides useful over a very wide wavelength range from the ultraviolet to infrared," Appl. Phys. Lett., vol. 77, no. 19, pp. 2961-2963, Nov. 6, 2000.

[6] L. Y. Chen, W. S. Tsai, W. H. Hsu, K. Y. Chen, and W. S. Wang, "Fabrication and characterization of benzocyclobutene optical waveguides by UV pulsed-laser illumination," IEEE J. Quantum Electron., vol. 43, no. 4, pp. 303-310, Apr. 2007.

[7] R. C. Alferness, R. V. Schmidt, and E. H. Turner, "Characteristics of Ti-diffused lithium niobate optical directional couplers," Appl. Opt., vol. 18, no. 23, pp. 4012-4016, Dec. 1979.

[8] L. B. Soldano and E. C. M. Pennings, "Optical multi-mode interference devices based on self-imaging: Principles and applications," J. Lightw. Technol., vol. 13, no. 4, pp. 615-627, Apr. 1995.

[9] M. H. Ibrahim, S.-Y. Lee, N. M. Kassim, and A. B. Mohammad, "Multimode interference optical splitter based on photodefinable benzocyclobutene (BCB 4024-40) polymer," Opt. Eng., vol. 46, no. 1, p. 013401, Jan. 2007. 